\title{
Cold Strangelets Formation with Finite Size Effects in High Energy Heavy-Ion Collisions *
}

\author{
Y.B. $\mathrm{He}^{1,2 \dagger}$, W.Q. Chao ${ }^{1,3,4}$, C.S. Gao ${ }^{1,2,4}$, and X.Q. $\mathrm{Li}^{1,4,5}$ \\ 1. China Center of Advanced Science and Technology (World Laboratory), \\ P.O. Box 8730, Beijing 100080, China \\ 2. Department of Physics, Peking University, Beijing 100871, China \\ 3. Institute of High Energy Physics, Academia Sinica, P.O. Box 918(4), Beijing 100039, China \\ 4. Institute of Theoretical Physics, Academia Sinica, P.O. Box 2735, Beijing 100080, China \\ 5. Department of Physics, Nankai University, Tianjin 300071, China
}

(October 22, 2018)

\begin{abstract}
We have studied the phase diagram and evolution of a strangelet in equilibrium with a finite hadronic gas. Significant finite size modifications of the phase diagram are found and their parameter dependences are studied. With the inclusion of finite size effects we have also been able to obtain the detailed properties of the cold strangelet emerging in the final stage of the isentropic expansion of a finite strange fireball in high energy heavy-ion collisions.
\end{abstract}

25.75.-q, 12.39.Ba, 21.65.+f, 24.85.+p

Typeset using REVTEX

* Partly supported by the National Natural Science Foundation of China

†E-mail: hyb@ccastb.ccast.ac.cn 


\section{INTRODUCTION}

It was speculated by Witten [1] that some of the dark matter in the universe might possibly exist in the form of strange quark matter (SQM), which consists approximately of the same amount of up, down and strange quarks, and might be formed after the big bang when the universe underwent the quark to hadron phase transition. Another place that people can expect to find SQM is in the "little bang", i.e. high energy heavy-ion collisions. It was supposed [2,3] that if a hot quark-gluon plasma (QGP) is formed after the collisions, the cooling process of the QGP might result in droplets of SQM, so called strangelets, which could serve as an unambiguous signature for the QGP formation. In fact, some heavy-ion experiments [4] are searching or planning to search for strangelets, hence reliable theoretical calculations of strangelets formation in heavy-ion collisions are in urgent need.

Recently Lee and Heinz [5] has presented a comprehensive study of the phase structure of a bulk strange quark matter in equilibrium with a bulk hadronic gas. Assuming Gibbs phase equilibrium and an isentropic expansion of the hot fireball, they found under some conditions strange quark matter can actually survive the hadronization and cooling process, giving rise to strangelet formation. On the other hand, taking into account the particle evaporation from the surface of the fireball, some rate calculations [3, 6] have been carried out to study the distillation and survival of strangelets in heavy-ion collisions. In this work we study finite size effects on strangelet formation, an important aspect which has been neglected in the above mentioned calculations.

It has been shown that finite size effects may strongly destabilize small strangelets at zero temperature [7], and significantly modify the properties of strangelets at finite temperature [8]. Finite size should be important as well in the study of the possible formation of strangelets, which are expected to be produced small in heavy-ion collisions [2, 3]. The present paper follows the work of Lee and Heinz [5] to study the phase diagram and evolution of strangelets in heavy-ion collisions, while extending it to include the essential finite size effects. We find that there are indeed significant finite size modifications of the phase dia- 
gram and evolution of strangelets. A realistic calculation that attempts to provide reliable information for the strangelets searches should therefore take finite size effects into account.

To study SQM in the context of the MIT bag model we have generally two ways to account for finite size effects. One employs the shell model, namely solving the Dirac equation with the bag boundary conditions and populating the energy levels of the bag [9, 3],10]. However, shell model calculations are very tedious even at zero temperature, and become extremely difficult when one tries to study the phase diagram and evolution. Another way to introduce finite size effects of strangelets is to explore a continuous density of states within the framework of multiple reflection expansion [11], which leads to the liquid drop model [7].12]. As shown by Madsen [7], the liquid drop model reproduces the overall structure of shell model results for strangelets, and allows much easier calculations than shell model. In this work we shall use the liquid drop model to incorporate finite size effects into the studies of phase diagram and evolution of strangelets.

This paper is organized as follows. In Sect. II we give a description of equations of state for the QGP and hadronic phase, with an emphasis on the inclusion of finite size effects in the QGP phase. In Sect. III we study the phase diagram of a strangelet in equilibrium with a finite hadronic phase, assuming the conservation of total baryon number and strangeness in the system. It is shown that the inclusion of finite size effects can modify the phase diagram in a significant way. The isentropic expansion of the system and possible formation of cold strangelets are explored in Sect. IV. We find that in contrast with bulk case the inclusion of finite size effects will give even more restrictive conditions under which dynamical formation of a cold strangelet is possible. We summarize our work in Sect. $\mathrm{\nabla}$.

\section{EQUATION OF STATE}




\section{A. The QGP Phase}

We consider the QGP phase as a gas of free massless up and down quarks $\left(m_{u}=m_{d}=0\right)$, massive strange quarks $\left(m_{s}=150 \mathrm{MeV}\right)$, their antiquarks and gluons confined in an MIT bag. In the multiple reflection expansion of Balian and Bloch [11] the density of states for particle species $i$ present in the bag is given by,

$$
\frac{d N_{i}}{d k}=g_{i}\left\{\frac{1}{2 \pi^{2}} k^{2} V^{Q}+f_{S}^{(i)}\left(\frac{m_{i}}{k}\right) k S^{Q}+f_{C}^{(i)}\left(\frac{m_{i}}{k}\right) C^{Q}+\ldots\right\}
$$

For spherical QGP droplets $V^{Q}=4 \pi\left(R^{Q}\right)^{3} / 3$ is the volume of the bag, $S^{Q}=4 \pi\left(R^{Q}\right)^{2}$ is the surface area, and $C^{Q}=8 \pi R^{Q}$ is the extrinsic curvature of the bag surface. The factor $g_{i}$ is the statistical weight (6 for quarks and antiquarks, and 16 for gluons).

The functions $f_{S}^{(i)}$ and $f_{C}^{(i)}$ are surface and curvature terms for particle species $i$. The surface term for quarks was given by Berger and Jaffe [13] as

$$
f_{S}^{(q)}\left(\frac{m_{q}}{k}\right)=-\frac{1}{8 \pi}\left\{1-\frac{2}{\pi} \arctan \frac{k}{m_{q}}\right\} .
$$

In particular, the surface term for massless quarks and gluons is zero. The curvature term

for gluons is $f_{C}^{(g)}=-1 / 6 \pi^{2}$ [14, while for massless quarks $f_{C}^{(q)}(0)=-1 / 24 \pi^{2}$ [15]. It has been shown by Madsen [0] that the following ansatz works for the curvature term for massive quarks:

$$
f_{C}^{(q)}\left(\frac{m_{q}}{k}\right)=\frac{1}{12 \pi^{2}}\left\{1-\frac{3 k}{2 m_{q}}\left(\frac{\pi}{2}-\arctan \frac{k}{m_{q}}\right)\right\}
$$

The thermodynamic potential of the QGP phase can be derived from,

$$
\Omega=\sum_{i} \Omega_{i}+B V^{Q}
$$

where $B$ is the bag constant, and the thermodynamic potential of particle species $i$ can be written as

$$
\begin{aligned}
\Omega_{i} & =\mp T \int_{0}^{\infty} d k \frac{d N_{i}}{d k} \ln \left[1 \pm \exp \left(-\left(E_{i}(k)-\mu_{i}\right) / T\right)\right] \\
& =\Omega_{i, V} V^{Q}+\Omega_{i, S} S^{Q}+\Omega_{i, C} C^{Q}
\end{aligned}
$$


with the upper sign for fermions and the lower for bosons. Here $\mu_{i}$ is the chemical potential. We have assumed isospin symmetry so that $\mu_{u}=\mu_{d}=\mu_{q}$, and there are two independent chemical potentials in the QGP phase, namely $\mu_{q}$ and $\mu_{s}$. To get an impression of the finite size effects introduced here, we have plotted in Fig. 1 the temperature dependence of individual surface and curvature contributions of particle species to the thermodynamic potential. One sees from Fig. 1 that they are all increasing functions of the temperature. For a baryon and strangeness free QGP droplet (i.e. $\mu_{q}=\mu_{s}=0$ ) the surface and curvature contributions vanish at zero temperature (Fig. 1(a) and (b)), while for nonvanishing chemical potentials there are finite contributions even at zero temperature. We see that significant finite size effects can be given by an approach of multiple reflection expansion, which can be relevant in the study of QGP phase structure.

After the construction of the thermodynamic potential, we can readily obtain the thermodynamic quantities of the system as follows. The number of quarks can be derived from

$$
N_{i}^{Q}=-\left(\frac{\partial \Omega_{i}}{\partial \mu_{i}}\right)_{T, V^{Q}},
$$

and

$$
N_{b}^{Q}=\frac{1}{3} \sum_{i} N_{i}
$$

gives the total baryon number of the QGP droplet. The total entropy of the QGP phase is

$$
S^{Q}=-\left(\frac{\partial \Omega}{\partial T}\right)_{V^{Q}, \mu_{i}} .
$$

\section{B. The Hadronic Phase}

Now we consider the equation of state for the hadronic phase. We model the hadronic phase as a mixture of Bose-Einstein and Fermi-Dirac gases of mesons $\pi, K, \eta$, baryons $N, \Lambda, \Sigma, \Xi, \Omega, \Delta$ and their anti-particles [16,12]. We will show below that using this limited particle spectrum gives practically identical results compared with the case in which a spectrum of all hadrons up to $M \sim 2 \mathrm{GeV}$ is used, thus sufficient for the study of the phase 
diagram. We can write down the pressure, particle number, and energy density expressions for pointlike hadron species $i$ as follows,

$$
\begin{aligned}
P_{i}^{p t} & =\frac{g_{i}}{6 \pi^{2}} \int_{0}^{\infty} d k \frac{k^{4}}{E_{i}(k)} \frac{1}{e^{\left(E_{i}(k)-\mu_{i}\right) / T} \pm 1} \\
N_{i}^{p t} & =\frac{g_{i} V^{H}}{2 \pi^{2}} \int_{0}^{\infty} d k \frac{k^{2}}{e^{\left(E_{i}(k)-\mu_{i}\right) / T} \pm 1} \\
\varepsilon_{i}^{p t} & =\frac{g_{i}}{2 \pi^{2}} \int_{0}^{\infty} d k \frac{k^{2} \varepsilon_{i}(k)}{e^{\left(E_{i}(k)-\mu_{i}\right) / T} \pm 1}
\end{aligned}
$$

where $g_{i}=(\operatorname{spin}) \times($ isospin $)$ is the degeneracy factor for each hadron, the \pm signs correspond to Fermi-Dirac and Bose-Eistein statistics, $\mu_{i}$ is the chemical potential of hadron species $i$ and given by the net numbers of light quark and strange quark, $\Delta N_{q}^{i}, \Delta N_{s}^{i}$, as

$$
\mu_{i}=\Delta N_{q}^{i} \mu_{q}^{H}+\Delta N_{s}^{i} \mu_{s}^{H}
$$

if chemical equilibriums are assumed.

The thermodynamic quantities for the hadronic gas are corrected by a Hagedorn eigenvolume factor, $\left(1+\varepsilon^{p t} / 4 B\right)^{-1}$, to take into account the repulsive interactions between the hadrons [17, 16], which is essential for the existence of phase transition in the considered model,

$$
\begin{aligned}
P^{H} & =\frac{1}{1+\varepsilon^{p t} / 4 B} \sum_{i} P_{i}^{p t}, \\
N_{b}^{H} & =\frac{1}{1+\varepsilon^{p t} / 4 B} \sum_{i} b_{i} N_{i}^{p t}, \\
N_{s}^{H} & =\frac{1}{1+\varepsilon^{p t} / 4 B} \sum_{i} s_{i} N_{i}^{p t},
\end{aligned}
$$

where $\varepsilon^{p t}=\sum_{i} \varepsilon_{i}^{p t}$, and $b_{i}, s_{i}$ are the baryon number, strange valence quark number of hadron species $i$ respectively.

We want to emphasize that a finite hadronic phase, instead of a bulk one [12], is considered in this work. In the case of a bulk QGP phase in equilibrium with a bulk hadronic phase, the conservation of total net baryon number and strangeness in the system is expressed in terms of a fixed strangeness fraction, namely the net number of strange quarks per baryon. In our work, however, to include the finite size effects we have considered a finite 
QGP phase, whose volume, baryon number and strangeness have been specified. Therefore, if the system consisting of a QGP phase and a hadronic phase is assumed to conserve total net baryon number and strangeness as a whole (the conservation of total net baryon number and strangeness will be discussed furthermore below), the volume of the hadronic phase has to be specified as well in order to conserve the total net baryon number and strangeness in a consistent way. On the other hand, we have not included surface and curvature contributions from hadrons in our calculations since we have not found appropriate expressions for them [18]. Nevertheless, there were expections that these contributions from hadrons may be less important compared with those from quarks and gluons 19,20.

\section{PHASE DIAGRAM WITH FINITE SIZE EFFECTS}

We start this section with a description of our scenario. If a baryon-rich QGP is formed in high energy heavy-ion collisions with an equal number of $s$ and $\bar{s}$ quarks, the system will suffer a strangeness enrichment due to the early black-body radiation of more kaons $(q \bar{s})$ than anti-kaons $(\bar{q} s)$ off the fireball, and then the system reaches the phase coexistence region [3]. We shall study the phase diagram (this section) and further phase evolution (next section) of the resulting system with finite net strangeness.

Following the work of Lee and Heinz [5] we consider a quasistationary phase transition between the QGP and hadronic gas, which assumes that the thermodynamic equilibrium time as well as the hadronization time are small compared to the lifetime of the mixed phase so that a complete thermodynamic equilibrium can be held during the whole phase transition. In this work we have not taken into account the effects of particle evaporation off the system which may change the total net baryon number, strangeness content of the system [3,6]. As a result, the total net baryon number in the system is kept a constant. Furthermore, since we expect that the typical time scales in heavy-ion collisions are too short to establish a flavor equilibrium due to the weak interactions which can convert strange quarks to nonstrange quarks and vice versa, it is assumed that the total net strangeness in 
the system is conserved. Note that the total net baryon number and strangeness can be simultaneously conserved in a consistent way only when a finite hadronic phase is considered in connection with a finite QGP phase, as we discussed in Sect. П.

The Gibbs equilibrium between the QGP and hadronic phase is given by $P^{Q}=P^{H}$ (mechanical equilibrium), $T^{Q}=T^{H}$ (thermal equilibrium), $\mu_{q}^{Q}=\mu_{q}^{H}, \mu_{s}^{Q}=\mu_{s}^{H}$ (chemical equilibrium). Taking generally the temperatures and chemical potentials in the two phases equal, we are left with the pressure balance between the two phases,

$$
P^{Q}\left(T, \mu_{q}, \mu_{s} ; R^{Q}\right)=P^{H}\left(T, \mu_{q}, \mu_{s}\right)
$$

Note that the pressure generated by the QGP phase is a function of the QGP droplet radius $R^{Q}$, since we have included finite size effects into the equation of state for the QGP phase.

The conservation of total net baryon number and strangeness in the system gives two constraints,

$$
N_{b}^{t o t}=N_{b}^{Q}\left(T, \mu_{q}, \mu_{s} ; R^{Q}\right)+N_{b}^{H}\left(T, \mu_{q}, \mu_{s} ; V^{H}\right)
$$

and

$$
N_{s}^{t o t}=N_{s}^{Q}\left(T, \mu_{q}, \mu_{s} ; R^{Q}\right)+N_{s}^{H}\left(T, \mu_{q}, \mu_{s} ; V^{H}\right)
$$

where the volume of the hadronic phase is given by

$$
V^{H}=V^{t o t}-V^{Q}
$$

The phase diagram of the system is determined by Eqs. (16) - (19), and shown in Fig. 2(a) for parameters $B^{1 / 4}=180 \mathrm{MeV}, m_{s}=150 \mathrm{MeV}$, and a fixed strangeness fraction (namely net number of strange quarks per baryon) $f_{s}=0.5$. Full lines in Fig. 2(a) illustrate our results for a finite system with total net baryon number $N_{b}^{\text {tot }}=100$ and strange quark number $N_{s}^{t o t}=50$. To be more specific, the full line separating the pure QGP phase and mixed phase (MP) is obtained by setting $V^{H}=0$ in Eqs. (17) and (18), and solving Eqs. (16) -(18) for $\left(\mu_{q}, \mu_{s}, R^{Q}\right)$ at a given $T, N_{b}^{\text {tot }}$ and $N_{s}^{\text {tot }}$. On the other hand, drawing the boundary 
between the mixed phase (MP) and hadronic gas (HG) is a little more delicate. For a given value of $V^{\text {tot }}$, we solve Eqs. (16) -(19) for $\left(\mu_{q}, \mu_{s}, R^{Q}, V^{H}\right)$ at fixed $T, N_{b}^{t o t}$ and $N_{s}^{\text {tot }}$. We then increase the value of $V^{\text {tot }}$ until $V^{\text {tot }}$ is so large that Eqs. (16)-(19) have no solutions for $\left(\mu_{q}, \mu_{s}, R^{Q}, V^{H}\right)$, which implies that the QGP and hadronic phase can no longer coexist in equilibrium, and thus we have reached the boundary between the mixed phase (MP) and hadronic gas $(\mathrm{HG})$. We observe that at the MP-HG boundary the QGP droplet may have non-vanishing baryon number and strangeness, which is of course a consequence of the inclusion of finite size effects. To check the consistency of this solution procedure, we have also tried to decrease the value of $V^{\text {tot }}$ while solving Eqs. (16) - (19) until no solutions exist, and found that we exactly arrived at the QGP-MP boundary, which we have obtained above.

In comparison with the above case of a finite system, the dashed curves in Fig. 2(a) show the phase diagram of a bulk QGP in equilibrium with a bulk hadronic phase, i.e. without finite size effects, which is identical to the results of Lee and Heinz in Fig. 6(c) of Ref. [5]. One sees from Fig. 2(a) that the hadronic phase grows significantly with the inclusion of finite size effects, while the QGP-MP boundary is little affected by the finite size corrections. We shall show in next section that this growth of the hadronic phase will greatly reduce the survival probabilities of cold strangelets which we wish to find in heavy-ion collisions.

The dotted lines in Fig. 2(a) is plotted for $N_{b}^{t o t}=50, N_{s}^{t o t}=25$, to show how the finite size modifications of phase diagram vary with the given $N_{b}^{t o t}$ and $N_{s}^{t o t}$, in comparison with the bulk case (dashed lines) and the case of $N_{b}^{t o t}=100$ and $N_{s}^{t o t}=50$ (full lines). It is of no surprise that with smaller $N_{b}^{\text {tot }}$ and $N_{s}^{\text {tot }}$ the smaller system has its phase diagram modified more prominently by the finite size effects.

To see how the modifications of phase diagram due to the inclusion of finite size effects are affected by the bag constant $B$, we have plotted in Fig. 2(b) and 2(c) the phase diagrams for $B^{1 / 4}=145 \mathrm{MeV}$ and $235 \mathrm{MeV}, f_{s}=0.5$, with all dashed lines for a bulk system, and all full lines for a finite system with total net baryon number $N_{b}^{\text {tot }}=100$, strange quark number $N_{s}^{\text {tot }}=50$. We see that finite size modifications are more significant for larger bag 
constants. This can be understood roughly that for given $N_{b}^{\text {tot }}, N_{s}^{\text {tot }}$, and $T$, larger bag constants generally correspond to more compact and smaller QGP droplets in equilibrium with a hadronic gas, and thus more important finite size effects. However, we want to point out that the full line in Fig. 2(c) separating the mixed phase (MP) and hadronic gas (HG) is somehow out of control at regions of high baryon number density $\rho_{b}$. This difficulty may come form two origins. First, at regions of high baryon number density the chemical potentials may become very large and lead to some numerical uncertainty in the relevant Bose-Einstein integrations, which can be solved by further careful examinations. Another possibility is that at these regions the radius $R^{Q}$ of QGP droplet in equilibrium with a finite hadronic gas tends to become so small $(\lesssim 1 \mathrm{fm})$ that the multiple reflection expansion in powers of $1 / R^{Q}$, which we have adopted to introduce finite size effects, could be somewhat dangerous, as previously warned by Madsen [7]. Fortunately, in our calculations this kind of disease sets in generally for very large bag constants. As it is expected that for sufficiently large bag constant strangelets can neither be stable nor metastable even at zero temperature and the formation of cold strangelets in heavy-ion collisions is very unlikely [3],6], therefore this problem will not become serious if we are only interested in the cases in which the formation of a cold strangelet can be possible.

\section{FORMATION OF COLD STRANGELETS}

In this section we turn to study the evolution of the system to see how a cold strangelet could be formed in the final stage of evolution and what its characteristics look like. While a full dynamical investigation of the fireball evolution is beyond the scope of this work, we assume that the system expands adiabatically, conserving its total entropy during the whole evolution [16,21]. Note again that we have not included the process of surface evaporation which may change the entropy, net baryon number and strangeness of the system. Therefore what we are exploring is a system that experiences a smooth hydrodynamic expansion,

conserving the total entropy $S^{t o t}$, net baryon number $N_{b}^{t o t}$, and strangeness $N_{s}^{t o t}$ [3]. As we 
have already described in Sect. III, the system considered here may have nonvanishing net strangeness due to the early surface radiation of hadrons after the collisions.

We first look at a QGP fireball which is expanding isentropically and cooling down until it reaches the boundary of mixed phase. The isentropic expansion trajectories in the pure QGP phase are determined by solving

$$
\begin{aligned}
& N_{b}^{t o t}=N_{b}^{Q}\left(T, \mu_{q}, \mu_{s} ; R^{Q}\right), \\
& N_{s}^{t o t}=N_{s}^{Q}\left(T, \mu_{q}, \mu_{s} ; R^{Q}\right),
\end{aligned}
$$

and

$$
S^{t o t}=S^{Q}\left(T, \mu_{q}, \mu_{s} ; R^{Q}\right)
$$

for $\left(T, \mu_{q}, \mu_{s}\right)$ at fixed $N_{b}^{t o t}, N_{s}^{t o t}, S^{t o t}$ and various $R^{Q}$. Increasing the value of $R^{Q}$ until the pressure balance Eq. (16) is possible, we will arrive at the boundary between the QGP and mixed phase, which we have obtained in the phase diagram given in last section.

Afterwards the isentropic expansion trajectories go into the mixed phase and are given by Eqs. (16) - (19), together with

$$
S^{t o t}=S^{Q}\left(T, \mu_{q}, \mu_{s} ; R^{Q}\right)+S^{H}\left(T, \mu_{q}, \mu_{s} ; V^{H}\right),
$$

which are solved for $\left(T, \mu_{q}, \mu_{s}, R^{Q}, V^{H}\right)$ at fixed $N_{b}^{t o t}, N_{s}^{t o t}, S^{t o t}$ and various $V^{\text {tot }}$. When we increase the value of $V^{\text {tot }}$ while solving these equations, two phenomena may be observed. One is that these equations have no solutions for $\left(T, \mu_{q}, \mu_{s}, R^{Q}, V^{H}\right)$ when $V^{\text {tot }}$ is large enough, which is found to be the very case in which the expansion trajectory hits the MP-HG boundary at some point. This observation further confirms the consistency of our treatment of finite size effects. In this case the expansion trajectory will then enter the hadronic phase, corresponding to a complete hadronization, and no cold strangelet could be formed. Another important phenomenon is that for $V^{t o t} \rightarrow \infty$ Eqs. (16)-(19) and (23) can always be solved at fixed $N_{b}^{t o t}, N_{s}^{t o t}$ and $S^{t o t}$, giving $T \rightarrow 0$. This implies that a cold strangelet could appear in the final stage of the evolution, which is of great interest in heavy-ion experiments. 
As manifestations of the above discussions, we show in Fig. 3 isentropic expansion trajectories of the system through the phase diagrams for $B^{1 / 4}=180 \mathrm{MeV}, N_{b}^{\text {tot }}=100$, and $N_{s}^{t o t}=0,50,100,200$, or strangeness fractions $f_{s}=0,0.5,1.0,2.0$. Our Fig. 3 can be compared with the results of Lee and Heinz for bulk phases given in Fig. 6 of Ref. [5] to see how the inclusion of finite size effects can modify the isentropic expansion trajectories of the system. It can be seen in Fig. 3 that for most cases the expanding system will hadronize completely. In particular, for $f_{s}=0.5$ and $S^{t o t} / N_{b}^{t o t}=5$ (Fig. 3(b)), in contrast with the bulk case (Fig. 6(c) in Ref. [5]) in which the system will always stay inside the mixed phase and give rise to cold strangelet formation, one finds that the inclusion of finite size effects tends to make the formation of cold strangelets even more difficult. In fact, with an impressive observation from Fig. 2(a) on how the phase diagram can be drastically modified by the finite size of the system, we can expect that currently existing calculations of possible formation of cold strangelets in heavy-ion collisions without finite size effects may have to become somehow less optimistic with the inclusion of finite size effects.

The inclusion of finite size effects may also affect in some way the strangeness separation process during the phase transition which is an essential ingredient for the possible formation of cold strangelets in heavy-ion collisions [3]. Fig. 4 shows the evolution details of the QGP droplet corresponding to the isentropic expansion trajectory for a system with zero net strangeness and $S^{t o t} / N_{b}^{t o t}=5$ in Fig. 3(a). In Fig. 4(a) the system experiences a cooling in the pure QGP phase, and reheating in the mixed phase which is mainly due to the energy surplus when quarks in the QGP are converted into hadrons in the hadronic gas. Fig. 4(b) shows a smooth variation of chemical potentials $\mu_{q}$ and $\mu_{s}$ during the phase transition. In particular, $\mu_{s}$ remains zero in the pure QGP phase whose net strangeness is zero, but increase continuously in the mixed phase even though the net strangeness of the system remains zero, indicating the occurrence of strangeness separation which can be seen also in Fig. 4(c) from a continuous increase of the strangeness fraction $f_{s}^{Q}$ of the QGP droplet. During the evolution in the mixed phase the net baryon number $N_{b}^{Q}$ (Fig. $\left.4(\mathrm{~d})\right)$ and radius $R^{Q}$ (Fig. $4(\mathrm{e})$ ) of the QGP droplet decrease monotonously. However, at the boundary between the mixed phase 
and hadronic gas $N_{b}^{Q}$ and $R^{Q}$ do not vanish, and only a rather moderate value of $f_{s}^{Q}$ can be reached. This is quite different from the corresponding cases for bulk phases studied previously [3,22], in which the QGP phase diminishes at the MP-HG phase boundary and the strangeness separation mechanism works in a much more effective way, leading to a much larger value of $f_{s}^{Q}$. In fact, as we have already known in the studies of phase diagrams that the hadronic phase grows on account of the mixed phase with the inclusion of the finite size effects, we can expect that the inclusion of the finite size effects tends to make the strangeness separation proceed to a less extent. Since the strangeness separation mechanism appears so important in calculations of the possible formation of cold strangelets in heavyion collisions, we hereby feel it a substantial task to include finite size effects in the future relevant investigations.

Now we study the formation of cold strangelets in heavy-ion collisions. The isentropic expansion trajectories in Fig. 3(c) and (d) indicate that for $S^{\text {tot }} / N_{b}^{t o t}=5$ the system will always stay in the mixed phase and expand infinitely to zero temperature and zero baryon number density, resulting in the formation of a cold strangelet. As an essential consequence of the inclusion of finite size effects, we can now obtain the properties of the surviving strangelet. For example, we list in Table. [ [ the properties of the resulting cold strangelet corresponding to the expansion trajectories for $S^{\text {tot }} / N_{b}^{t o t}=5$ and $f_{s}^{t o t}=1.0$ (Fig. 3(c)), 2.0 (Fig. 3(d)) respectively. Note in the case of $f_{s}^{t o t}=1.0$ all net strangeness is in the droplet $\left(N_{s}^{Q}=100\right)$, which is due to the fact that at $T \rightarrow 0$ chemical potentials $\mu_{q}, \mu_{s}$ in the phase equilibrium configuration acquire values such that only nucleons but no strange baryons are present in the hadronic gas.

However, the properties of cold strangelets given in Table. [ should not be taken too seriously for two reasons. First, an isentropic and equilibrium expansion of the fireball all the way to zero temperature assumed in this work is not a realistic scenario. Effects of particle evaporations off the surface of the fireball can be very important for the strangelet formation [3],6]. At sufficiently low temperature and density the QGP droplet may decouple 
from the surrounding hadronic gas and the thermodynamic equilibriums between them may be broken. On the other hand, the listed properties of the strangelets rely heavily on the parameters such as bag constant $B$, strange quark mass $m_{s}$, and on the given initial conditions of the system in terms of $N_{b}^{t o t}, N_{s}^{\text {tot }}$ and $S^{\text {tot }}$. Nevertheless, Table. If can give us a first impression of the characteristics of cold strangelets resulted from a simple dynamical evolution, and may provide clues for future construction of realistic dynamical evolution models.

The isentropic expansion trajectories in Fig. 3 imply that only for sufficiently low $S^{\text {tot }} / N_{b}^{\text {tot }}$ or large $f_{s}$ does the system allow the formation of cold strangelets. Following Lee and Heinz [5] we have plotted in Fig. 5 the maximum total entropy per baryon $S^{\text {tot }} / N_{b}^{\text {tot }}$ permitted by the formation of cold strangelets versus the strangeness fraction $f_{s}$, for two bag constants $B^{1 / 4}=145$ and $180 \mathrm{MeV}$, and a finite system with net baryon number $N_{b}^{\text {tot }}=100$. In comparison with the results for bulk phases in Fig. 8 of Ref. [5], we see that the inclusion of finite size effects give much more restrictive conditions under which cold strangelets could be formed. Even for bag constant as small as $B^{1 / 4}=145 \mathrm{MeV}$, which is expected to be the minimum value compatible with the usual nuclear matter phenomenology, and for strangeness fraction $f_{s}=3.0$, an upper limit $S^{\text {tot }} / N_{b}^{t o t} \lesssim 52$ is required to make the formation of cold strangelets possible. However, these results may depend on the net baryon number $N_{b}^{\text {tot }}$, or the size of the system, and are to be made more convincing by further detailed investigations.

\section{SUMMARY}

In the framework of multiple reflection expansion we have studied finite size effects on the phase diagram and evolution of a strangelet in equilibrium with a finite hadronic gas. Our aim is to check how important the finite size effects can be in the studies of possible formation of cold strangelets in high energy heavy-ion collisions. We have shown that there are indeed significant finite size modifications of the phase diagram, which are found to 
become more important for a system with larger bag constant $B$ or smaller total net baryon number $N_{b}^{\text {tot }}$.

Assuming an isentropic expansion of the system we have studied the finite size effects on the evolution of a strangelet. It is observed that the inclusion of finite size effects tends to make the strangeness separation mechanism less effective, and will give very restrictive conditions for the cold strangelet formation in heavy-ion collisions, expressed in terms of windows of the bag constant $B$, the total entropy per baryon $S^{\text {tot }} / N_{b}^{\text {tot }}$, and the strangeness fraction $f_{s}^{\text {tot }}$. With the inclusion of finite size effects we have been able to obtain quantitatively the properties of cold strangelets emerging in the final stage of the isentropic expansion of the system.

Even though the treatment of finite size effects within the framework of multiple reflection expansion may fail for very small strangelets as we have uncovered in the studies of the phase diagrams, and the isentropic and equilibrium evolution scenario studied in this work is not a realistic one, yet we can still conclude after this work that finite size effects do play an essential role and should be included in future realistic investigations of the possible formation of cold strangelets in high energy heavy-ion collisions.

\section{ACKNOWLEDGMENTS}

The authors would like to thank Prof. R. K. Su and Dr. S. Gao for helpful discussions. 


\section{REFERENCES}

[1] E. Witten, Phys. Rev. D 30, 272 (1984).

[2] H.-C. Liu and G. L. Shaw, Phys. Rev. D 30, 1137 (1984).

[3] C. Greiner, P. Koch and H. Stöcker, Phys. Rev. Lett. 58, 1825 (1987); C. Greiner, D. H. Rischke, H. Stöcker and P. Koch, Phys. Rev. D 38, 2797 (1988); C. Greiner, P. Koch and H. Stöcker, Phys. Rev. D 44, 3517 (1991).

[4] See, e.g., J. Barrette et al., Phys. Lett. B 252, 550 (1990); Phys. Rev. Lett. 70, 1763 (1993); A. Aoki et al., Phys. Rev. Lett. 69, 2345 (1992); K. Borer et al., Phys. Rev. Lett. 72, 1415 (1994). For a recent review see B. S. Kumar, Nucl. Phys. A590, 29c (1995).

[5] K. S. Lee and U. Heinz, Phys. Rev. D 47, 2068 (1993).

[6] H. W. Barz, B. L. Friman, J. Knoll and H. Schulz, Phys. Lett. B 242, 328 (1990).

[7] J. Madsen, Phys. Rev. Lett. 70, 391 (1993); Phys. Rev. D 47, 5156 (1993); ibid. 50, 3328 (1994).

[8] Y. B. He, C. S. Gao, X. Q. Li and W. Q. Chao, Phys. Rev. C 53, 1903 (1996).

[9] E. Farhi and R.L. Jaffe, Phys. Rev. D 30, 2379 (1984).

[10] E. P. Gilson and R. L. Jaffe, Phys. Rev. Lett. 71, 332 (1993).

[11] R. Balian and C. Bloch, Ann. Phys. 60, 401 (1970); T.H. Hansson and R.L. Jaffe, Ann. Phys. 151, 204 (1983).

[12] D. M. Jensen and J. Madsen, Proc. Strangeness and Quark Matter, Ed. G. Vassiliadis, A.D. Panagiotou, S. Kumar, and J. Madsen, World Scientific (1995), p.220.

[13] M. S. Berger and R. L. Jaffe, Phys. Rev. C 35, 213 (1987); 44, 566 (E) (1991).

[14] R. Balian and C. Bloch, Ann. Phys. 64, 271 (1970). 
[15] H.-T. Elze and W. Greiner, Phys. Lett. B 179, 385 (1986).

[16] U. Heinz, P. R. Subramanian, H. Stöcker and W. Greiner, J. Phys. G 12, 1237 (1986).

[17] R. Hagedorn and J. Rafelski, Phys. Lett. B 97, 180 (1980).

[18] For calculations of surface tension see, e.g., Z. Frei and A. Patkós, Phys. Lett. B 222, 469 (1989); ibid. 247, 381 (1990); B. Grossmann and M. L. Laursen, Nucl. Phys. B408, 637 (1993); A. A. Coley and T. Trappenberg, Phys. Rev. D 50, 4881 (1994); Y. Aoki and K. Kanaya, Phys. Rev. D 50, 6921 (1994).

[19] F. D. Mackie, Nucl. Phys. A245, 61 (1975).

[20] I. Mardor and B. Svetitsky, Phys. Rev. D 44, 878 (1991).

[21] H. Stöcker, G. Graebner, J. A. Maruhn and W. Greiner, Phys. Lett. B 95, 192 (1980); H. Stöcker and W. Greiner, Phys. Rep. 137, 277 (1986).

[22] U. Heinz, K. S. Lee and M. J. Rhoades-Brown, Mod. Phys. Lett. A 2, 153 (1987). 


\section{TABLES}

TABLE I. Properties of surviving strangelets for a system with $B^{1 / 4}=180 \mathrm{MeV}$, $m_{s}=150 \mathrm{MeV}, N_{b}^{\text {tot }}=100$, and $S^{\text {tot }} / N_{b}^{\text {tot }}=5$. The second column describes a cold strangelet resulting from the isentropic expansion trajectory $S^{\text {tot }} / N_{b}^{\text {tot }}=5$ in Fig. 3(c) with $N_{s}^{t o t}=100$ or equivalently $f_{s}^{\text {tot }}=1.0$, and the third column corresponds to the isentropic expansion trajectory $S^{t o t} / N_{b}^{\text {tot }}=5$ in Fig. $3(\mathrm{~d})$ with $N_{s}^{\text {tot }}=200$ or $f_{s}^{\text {tot }}=2.0$.

\begin{tabular}{ccc}
\hline \hline & $f_{s}^{t o t}=1.0$ & $f_{s}^{\text {tot }}=2.0$ \\
\hline$R^{Q}(\mathrm{fm})$ & 3.07 & 3.43 \\
$N_{b}^{Q}$ & 62.7 & 84.2 \\
$\rho_{b}^{Q}=N_{b}^{Q} / V^{Q}\left(\mathrm{fm}^{-3}\right)$ & 0.52 & 0.50 \\
$N_{s}^{Q}$ & 100. & 170. \\
$f_{s}^{Q}=N_{s}^{Q} / N_{b}^{Q}$ & 1.59 & 2.02 \\
$Z / N_{b}^{Q}=\left(1-f_{s}^{Q}\right) / 2$ & -0.30 & -0.51 \\
$E^{Q} / N_{b}^{Q}(\mathrm{MeV})$ & 1184 & 1250 \\
\hline \hline
\end{tabular}




\section{FIGURES}

FIG. 1. Temperature dependences of the surface and curvature contributions to the thermodynamic potential of light quarks ("q"), massive strange quark ("s"), and gluon ("g") for vanishing and nonvanishing chemical potentials. The "sum" in (b) and (d) stands for the total curvature contributions.

FIG. 2. (a) Phase diagrams for a QGP phase in equilibrium with a hadronic gas (HG), with bag constant $B^{1 / 4}=180 \mathrm{MeV}$ and fixed strangeness fraction $f_{s}=0.5$ : dashed lines for a bulk QGP and a bulk hadronic gas; full lines for a finite system with total net baryon number $N_{b}^{t o t}=100$ and net strange quark number $N_{s}^{\text {tot }}=50$; dotted lines for $N_{b}^{\text {tot }}=50$ and $N_{s}^{\text {tot }}=25$. Note that the pure QGP phase and the hadronic gas (HG) are separated by a finite mixed phase (MP). (b) Phase diagrams for a QGP phase in equilibrium with a hadronic gas (HG), with $B^{1 / 4}=145 \mathrm{MeV}$ and fixed $f_{s}=0.5$ : dashed lines for a bulk QGP and a bulk hadronic gas; full lines for a finite system with $N_{b}^{t o t}=100$ and $N_{s}^{t o t}=50$. (c) The same as in (b) but for $B^{1 / 4}=235 \mathrm{MeV}$.

FIG. 3. Isentropic expansion trajectories of a finite system with various total entropy per baryon $S^{\text {tot }} / N_{b}^{\text {tot }}$, total net baryon number $N_{b}^{\text {tot }}=100$, and net strange quark number (a) $N_{s}^{\text {tot }}=0$; (b) $N_{s}^{t o t}=50 ;(\mathrm{c}) N_{s}^{t o t}=100 ;(\mathrm{d}) N_{s}^{t o t}=200 .\left(B^{1 / 4}=180 \mathrm{MeV}, m_{s}=150 \mathrm{MeV}\right)$

FIG. 4. Variations of (a) temperature $T$, (b) chemical potentials $\mu_{q}, \mu_{s}$, (c) strangeness fraction $f_{s}^{Q}$, (d) net baryon number $N_{b}^{Q}$, and (e) radius $R^{Q}$ of the QGP droplet during the isentropic expansion of a system with $S^{t o t} / N_{b}^{t o t}=5, N_{b}^{t o t}=100$ and $N_{s}^{t o t}=0$ (corresponding to the isentropic expansion trajectory $S^{t o t} / N_{b}^{t o t}=5$ in Fig. $\left.3(\mathrm{a})\right) \cdot\left(B^{1 / 4}=180 \mathrm{MeV}, m_{s}=150 \mathrm{MeV}\right)$

FIG. 5. Maximum total entropy per baryon $S^{\text {tot }} / N_{b}^{t o t}$ permitted by the formation of cold strangelets as a function of the total strangeness fraction $f_{s}^{\text {tot }}$ for a system with $N_{b}^{\text {tot }}=100$ and two values of bag constant $B$. 


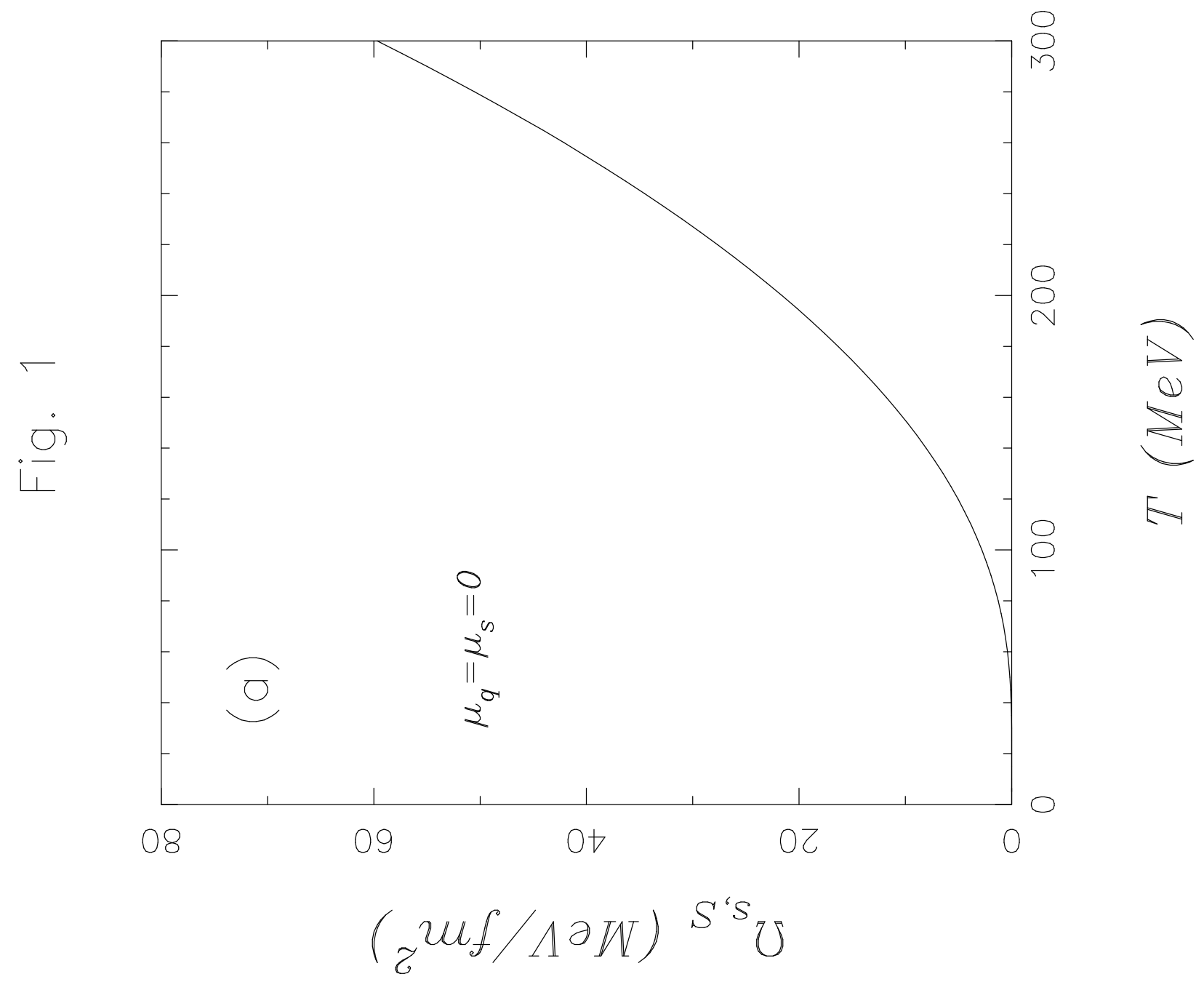

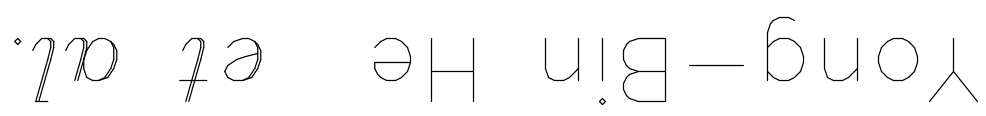




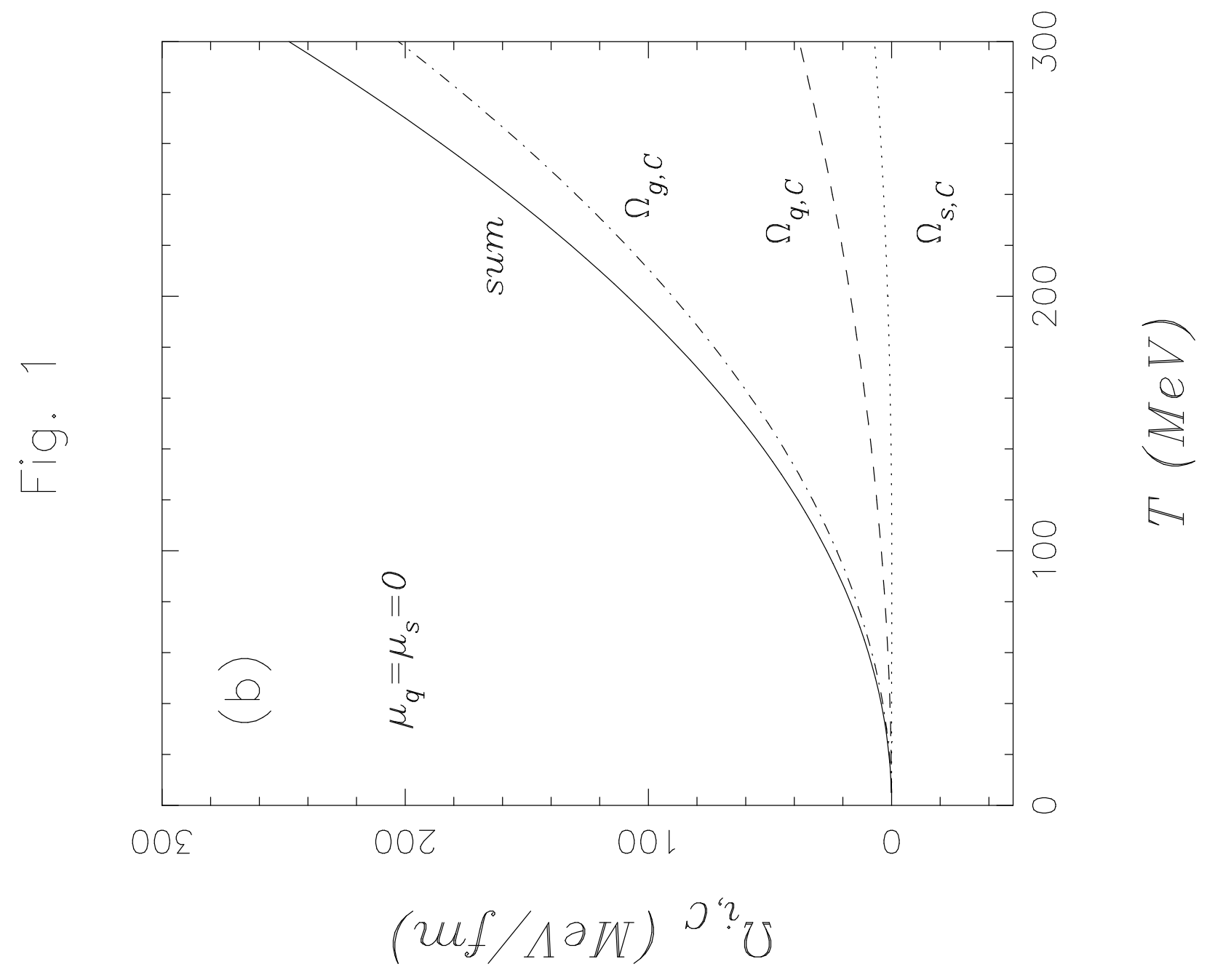

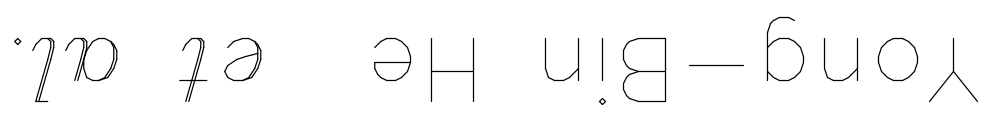




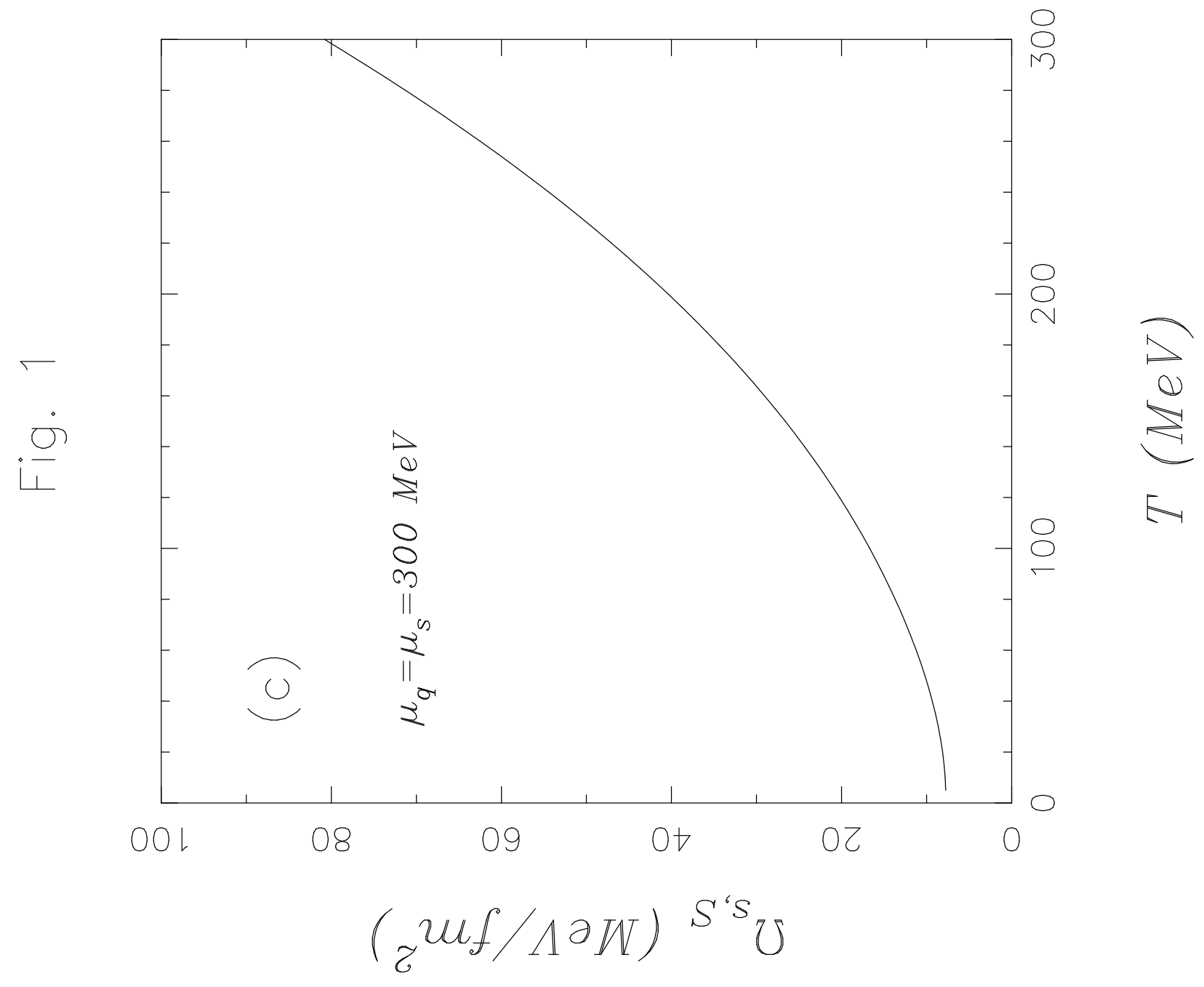

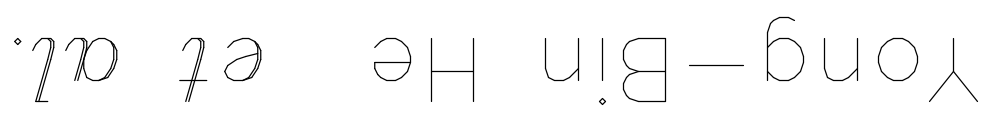




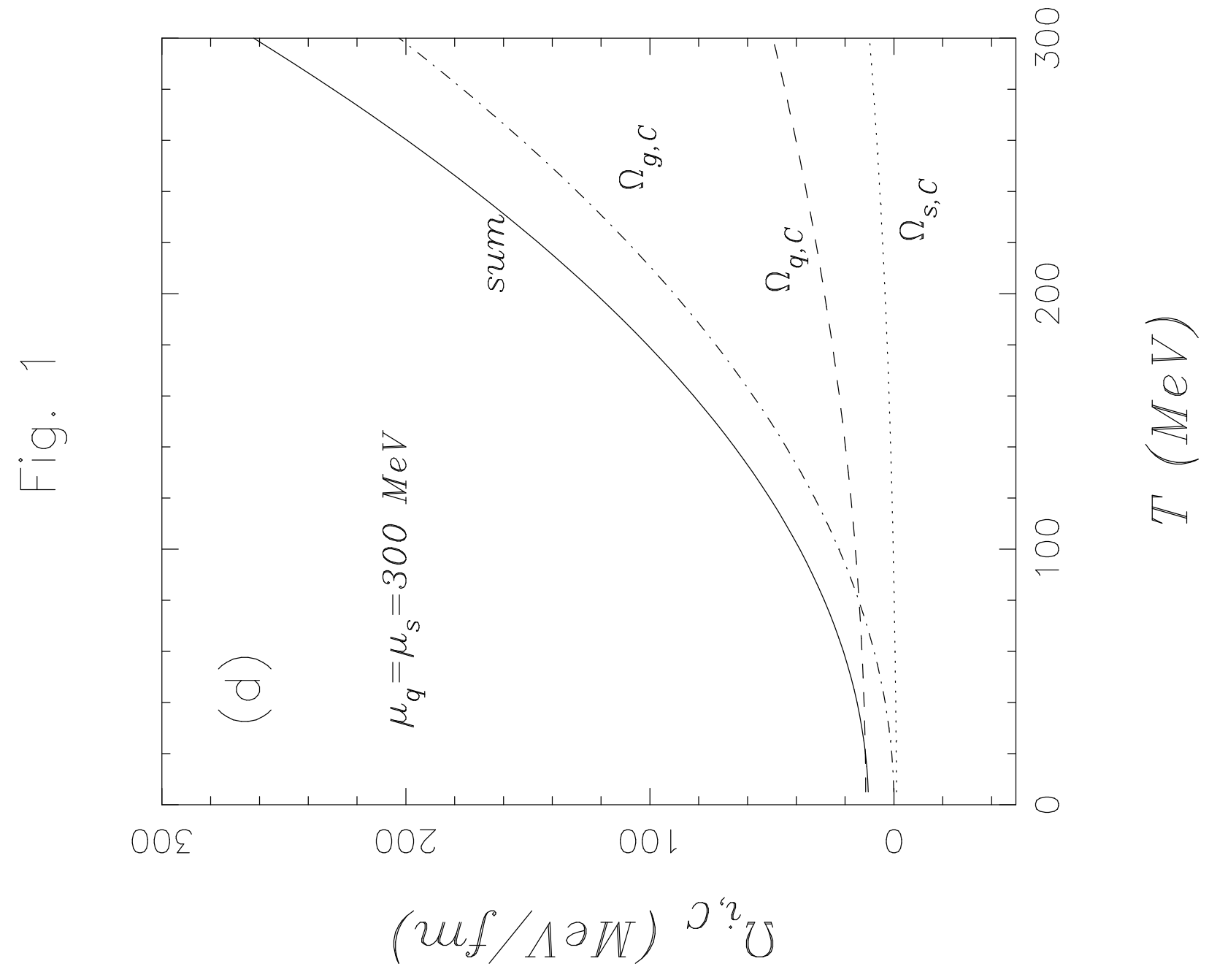

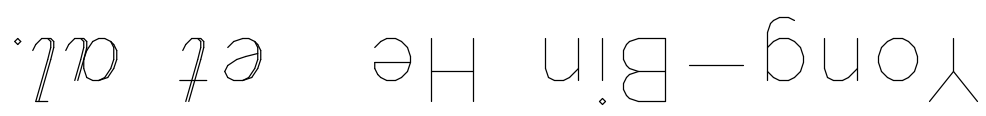




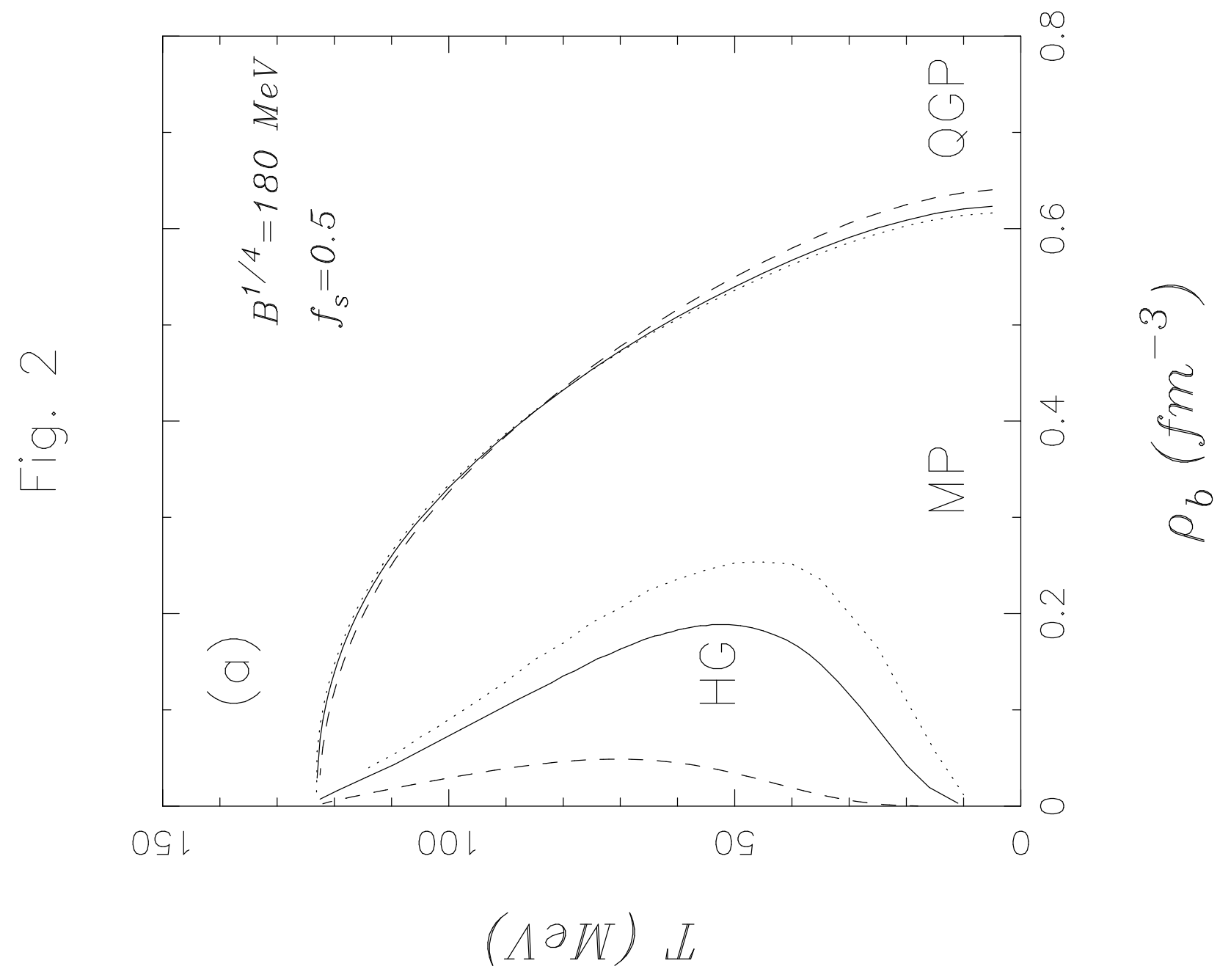

72 да ән แ!g-5นо人 


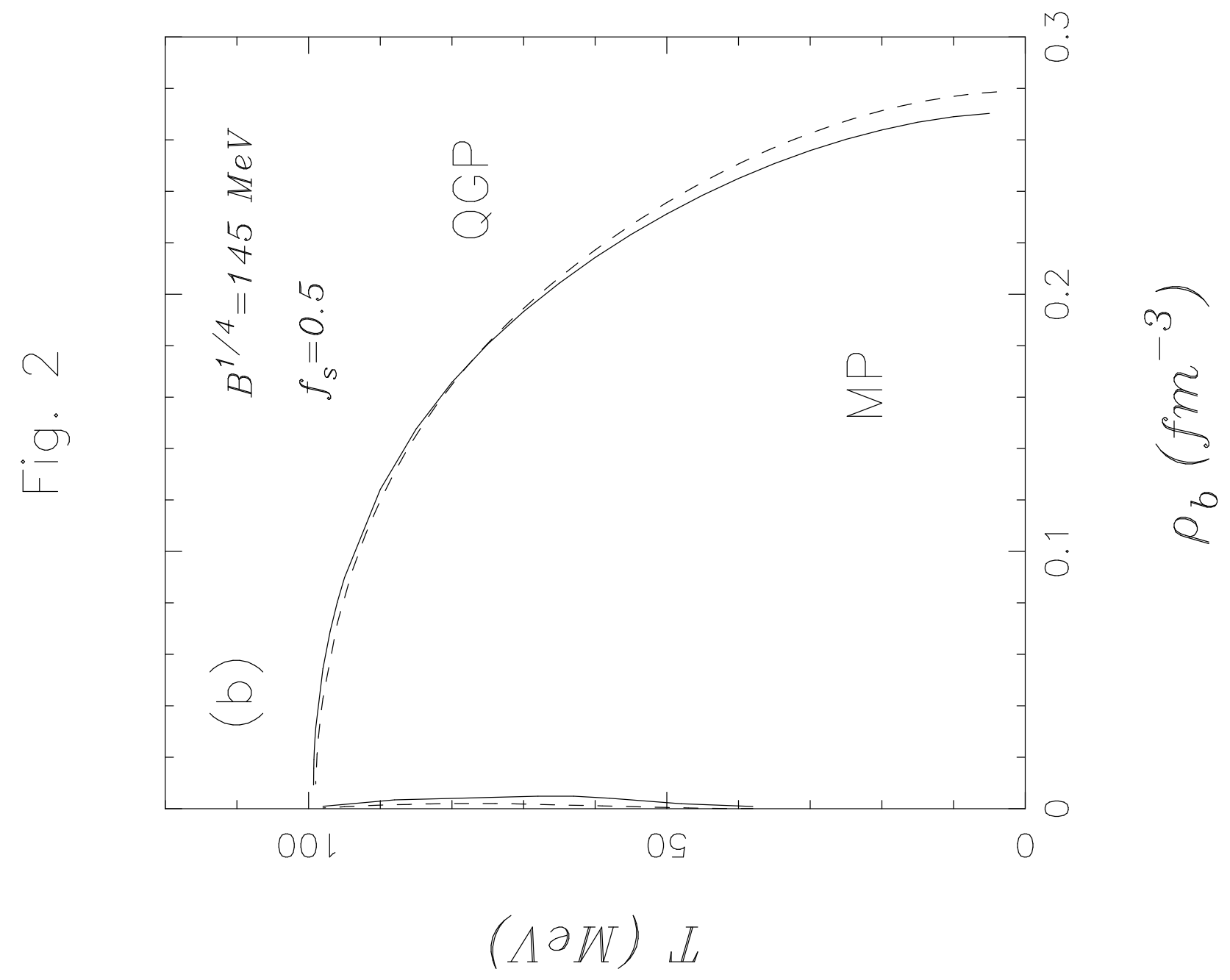

270 ға ән แ!g-бนо入 


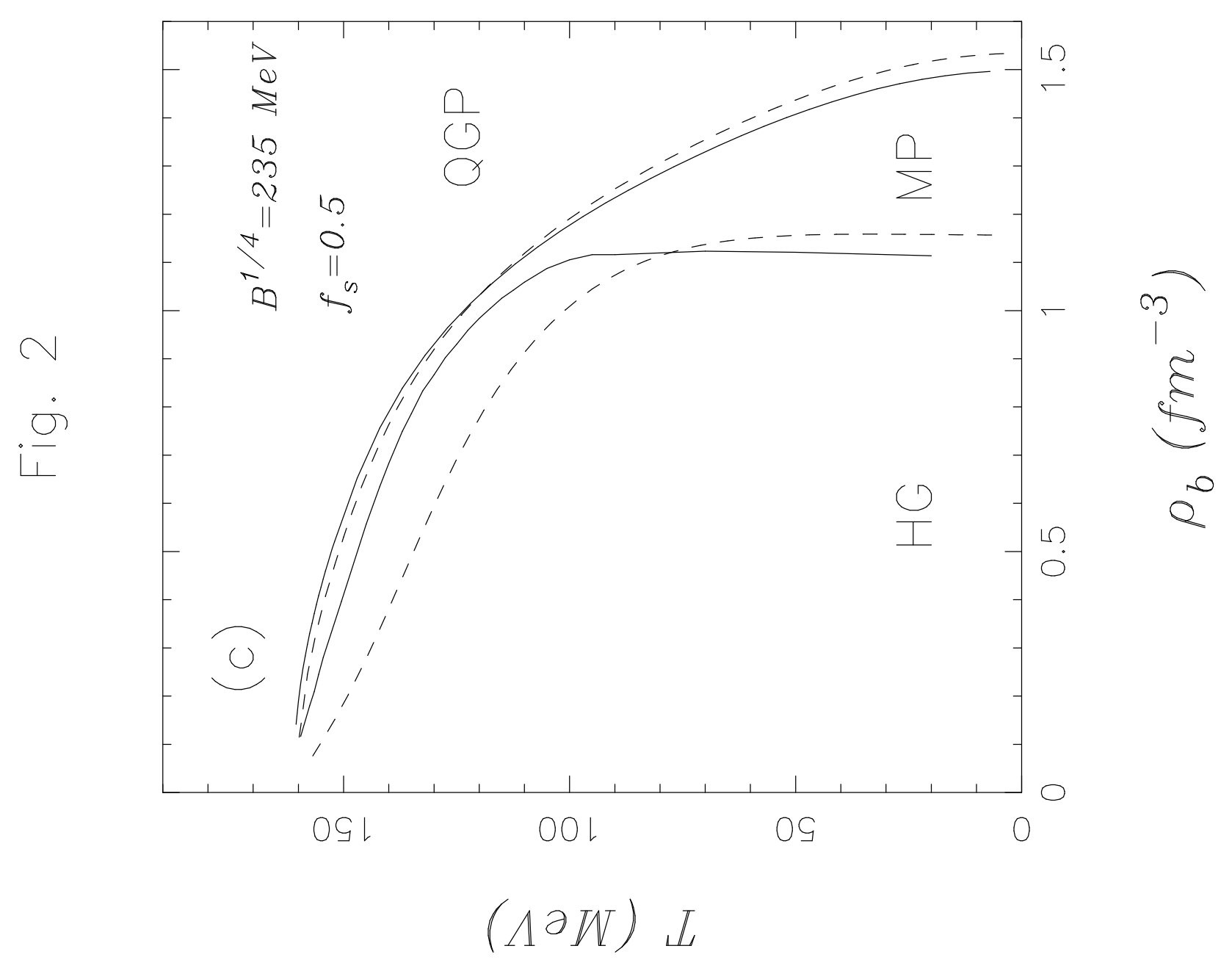

70 дә ән แ!g-6นо入 


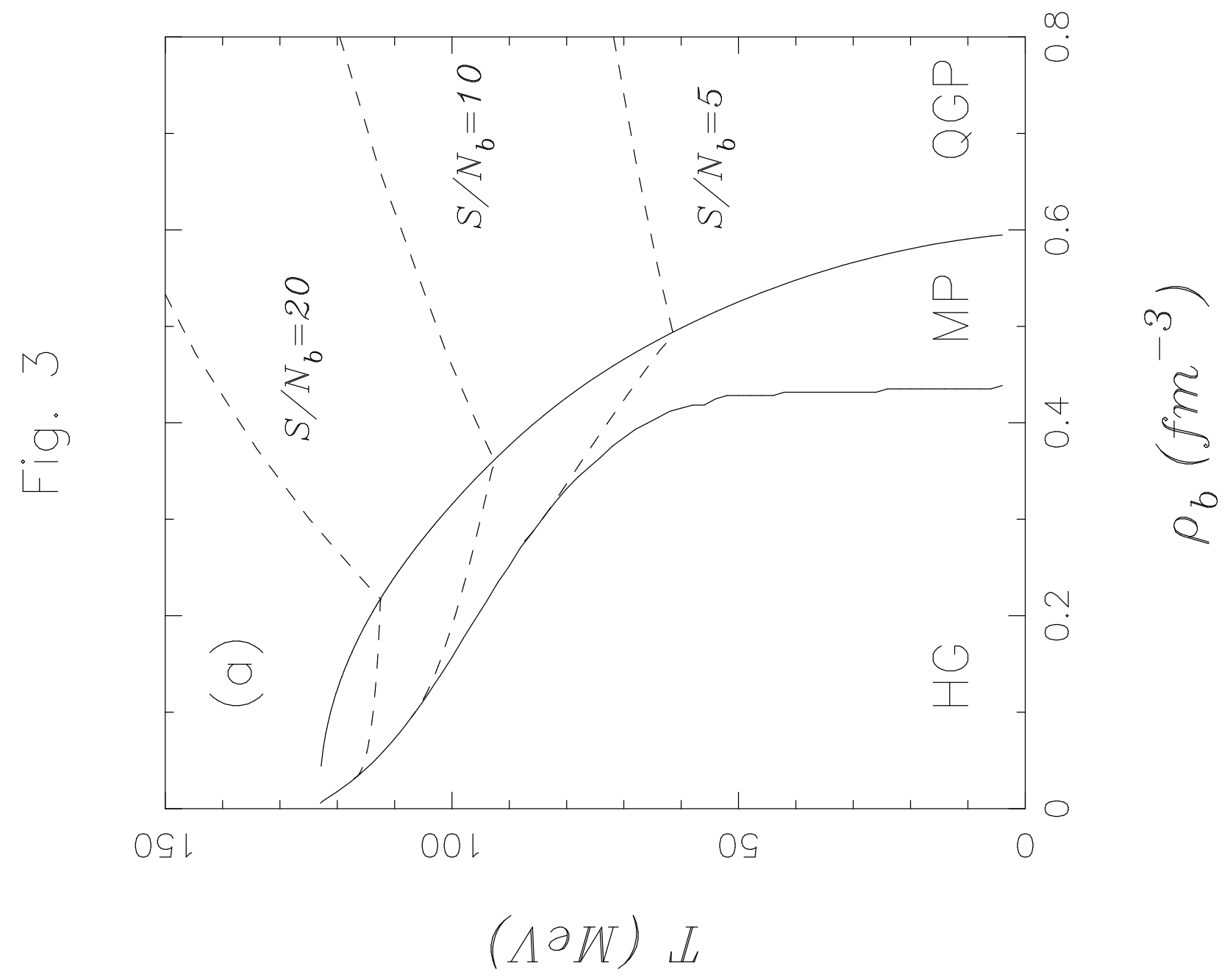

70 дә ән แ!g-6นо入 


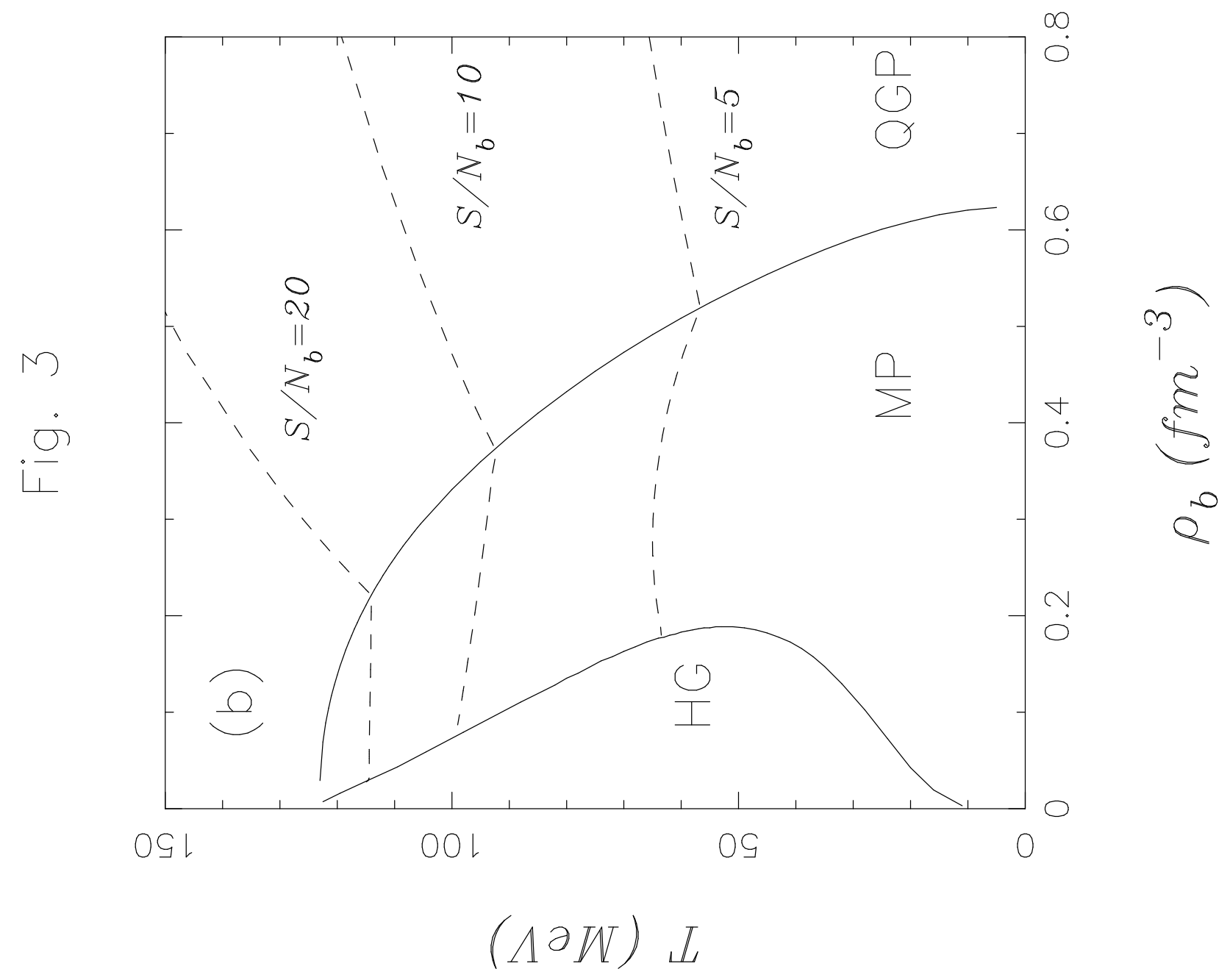

72 да ән แ!g-6นо人 


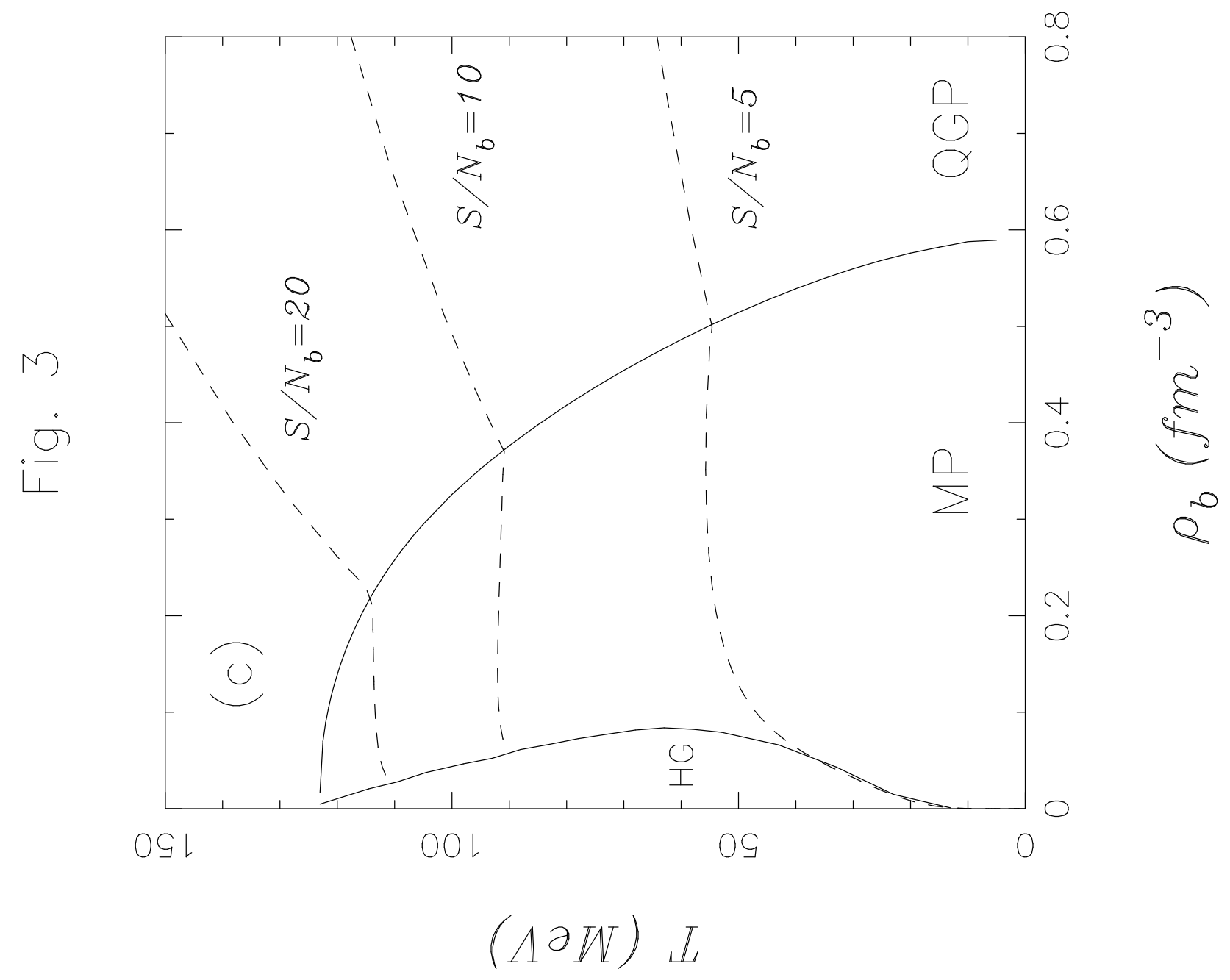

70 да ән u!g-6uо入 


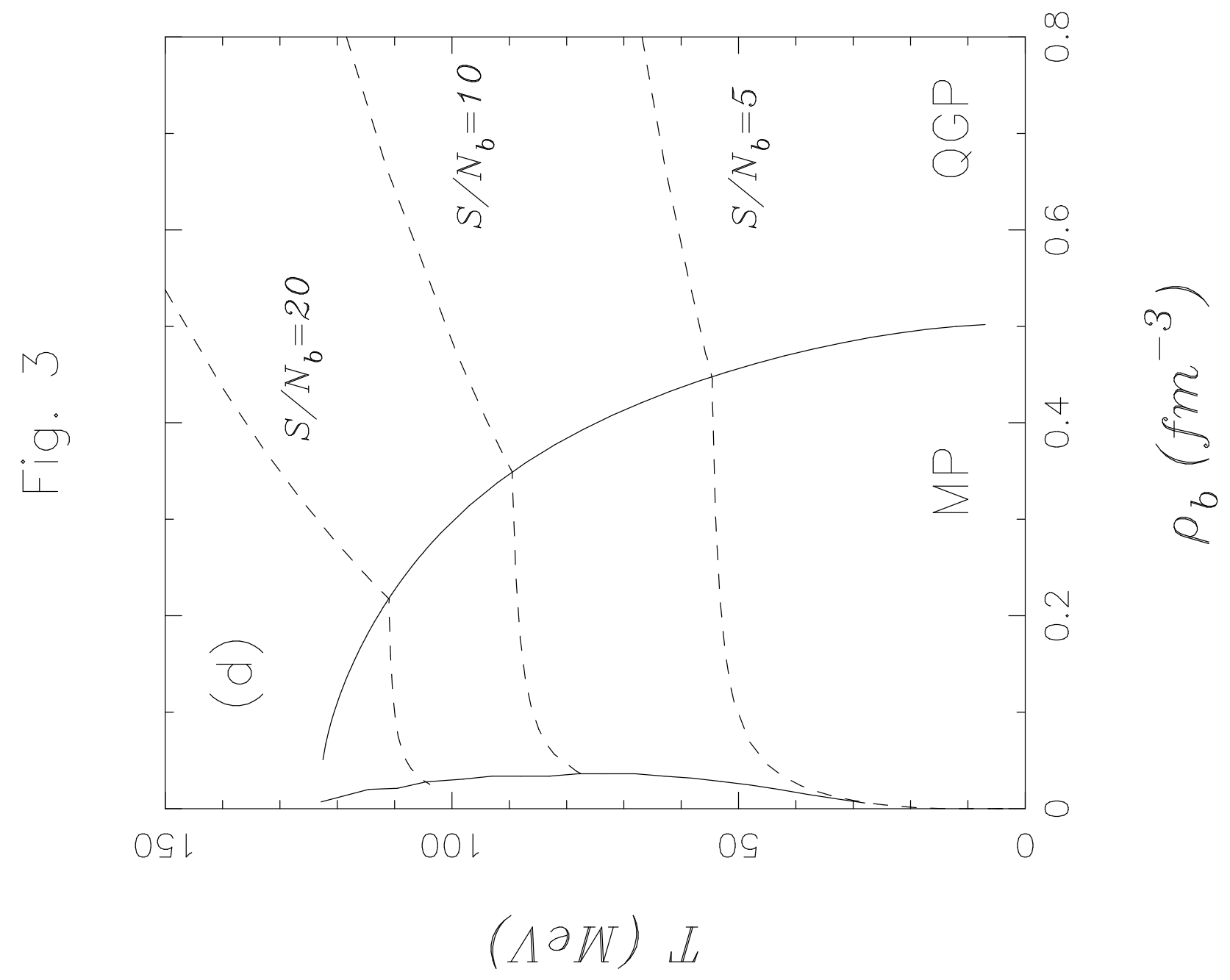

'70 ұа ән u!g-6uо入 


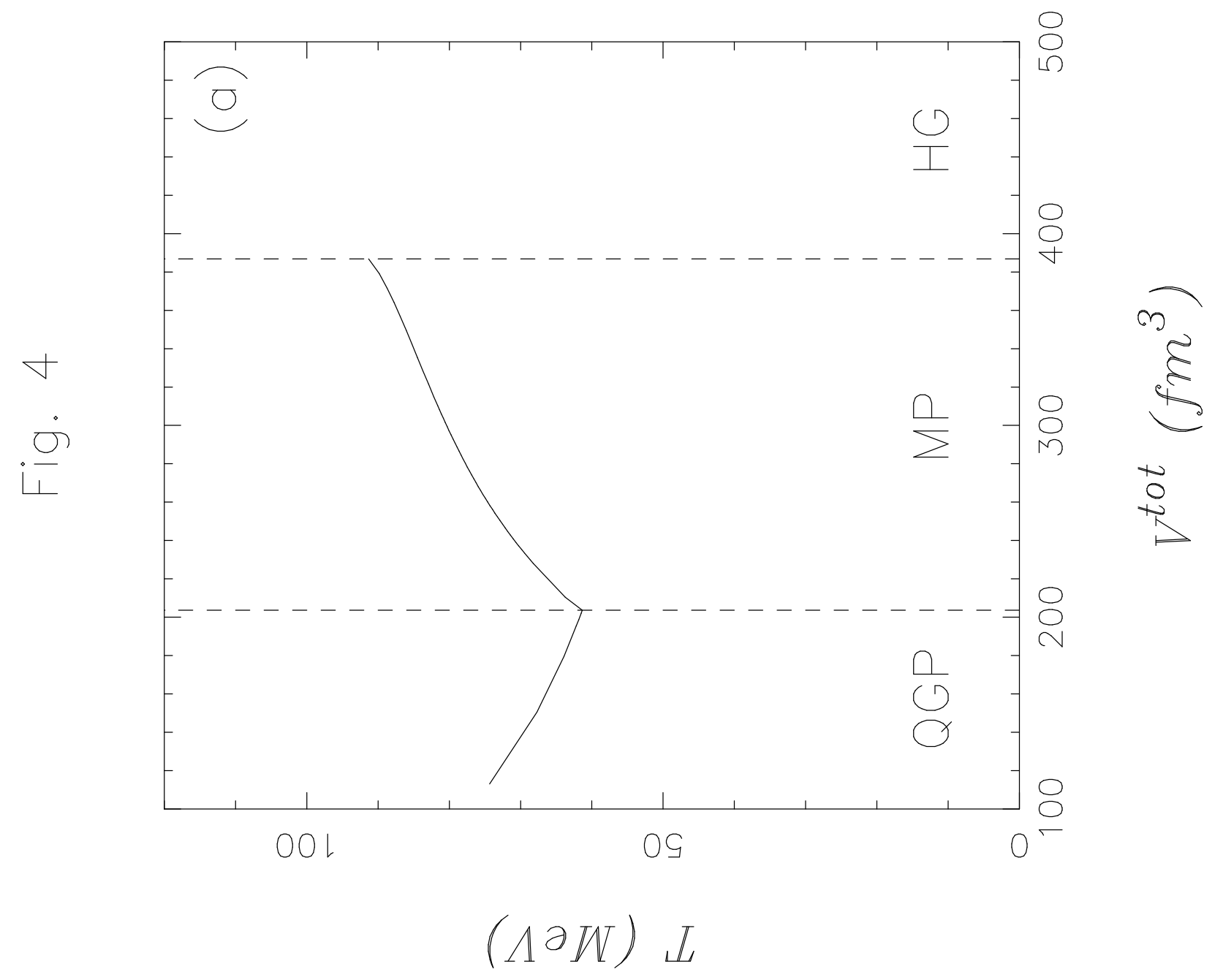

270 да ән щ!g-6นо人 


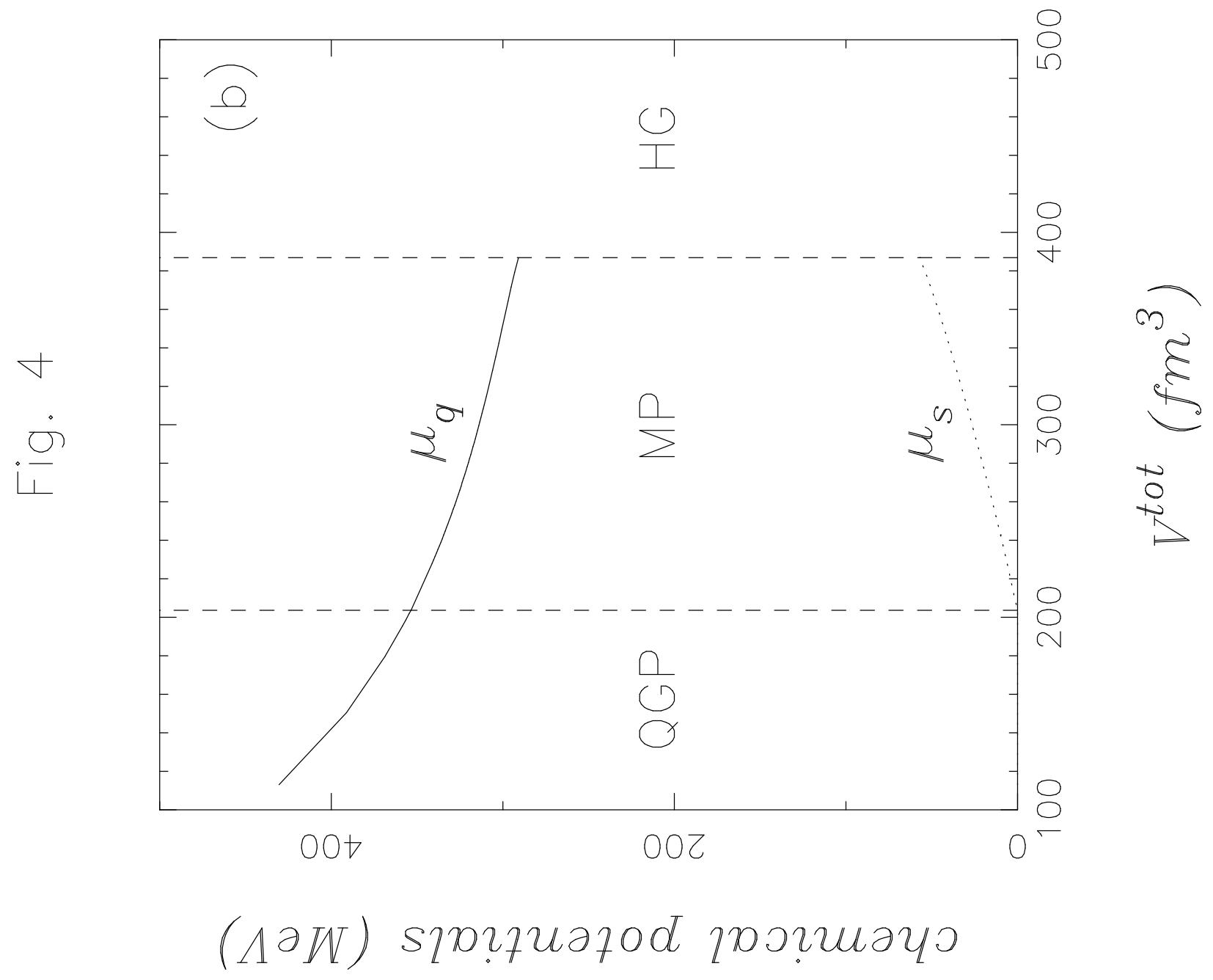

70 дә ән แ!g-6นо入 


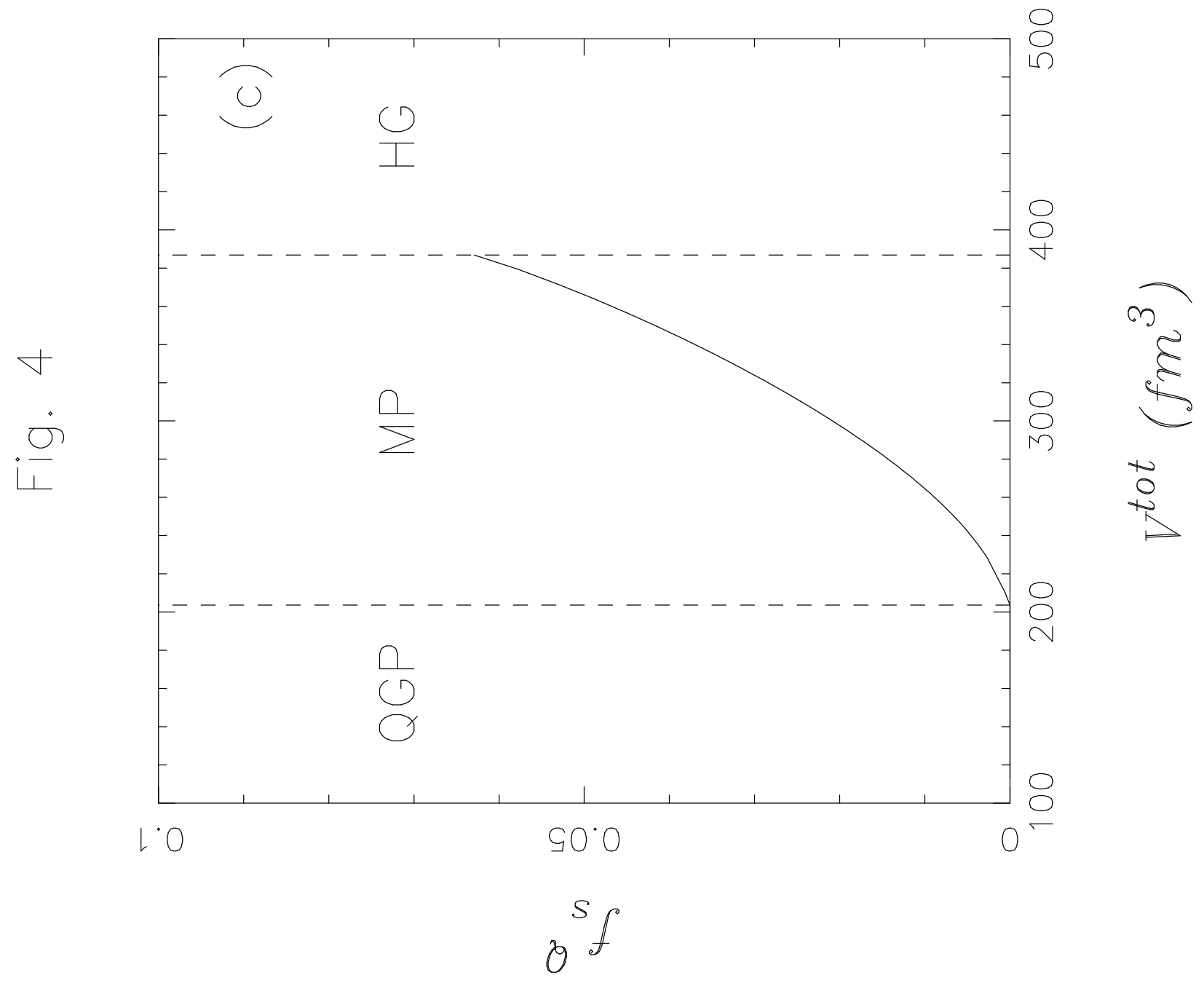

270 7ә $ә H$ u!g-5u०人 


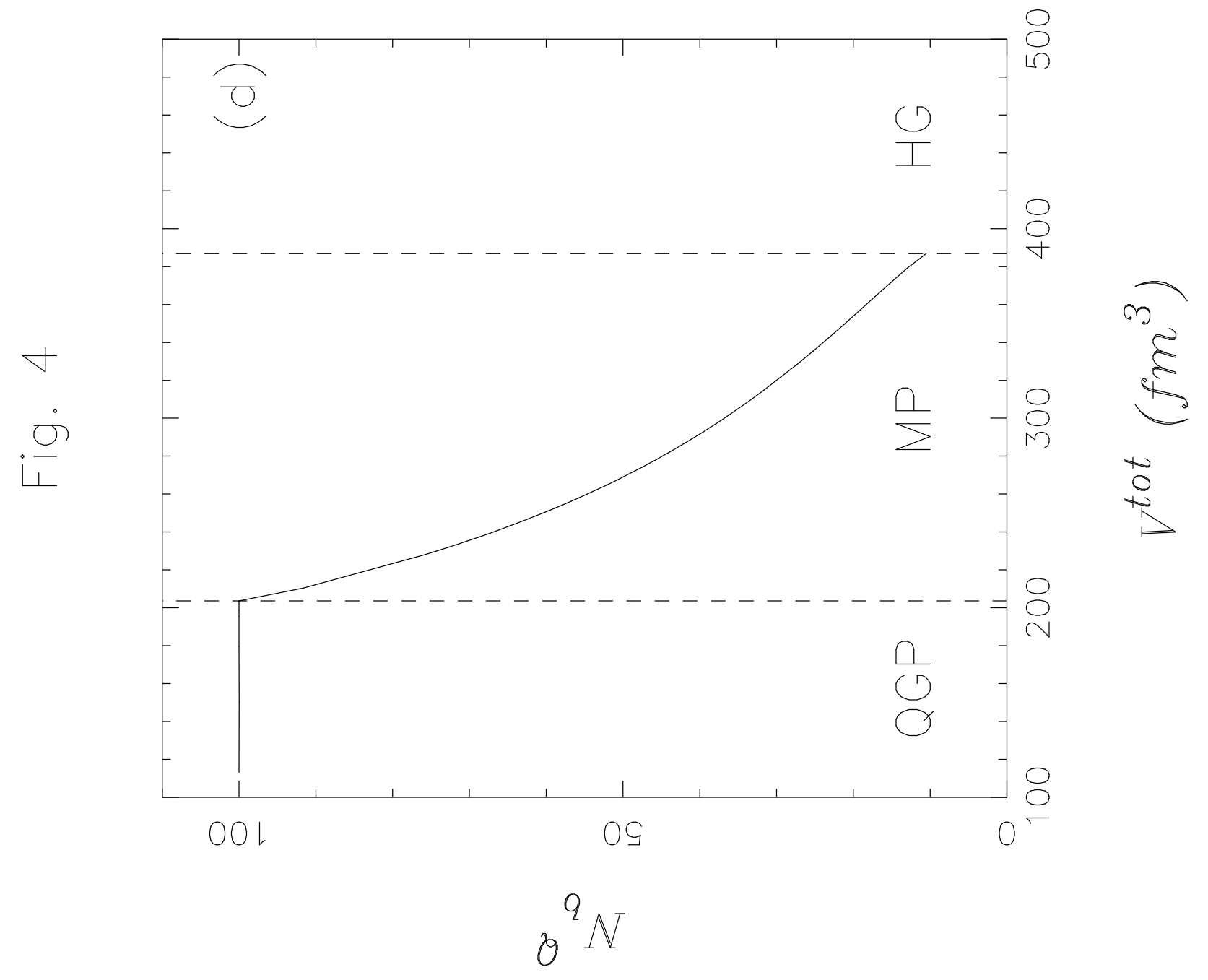

707 ว ән 4!. 


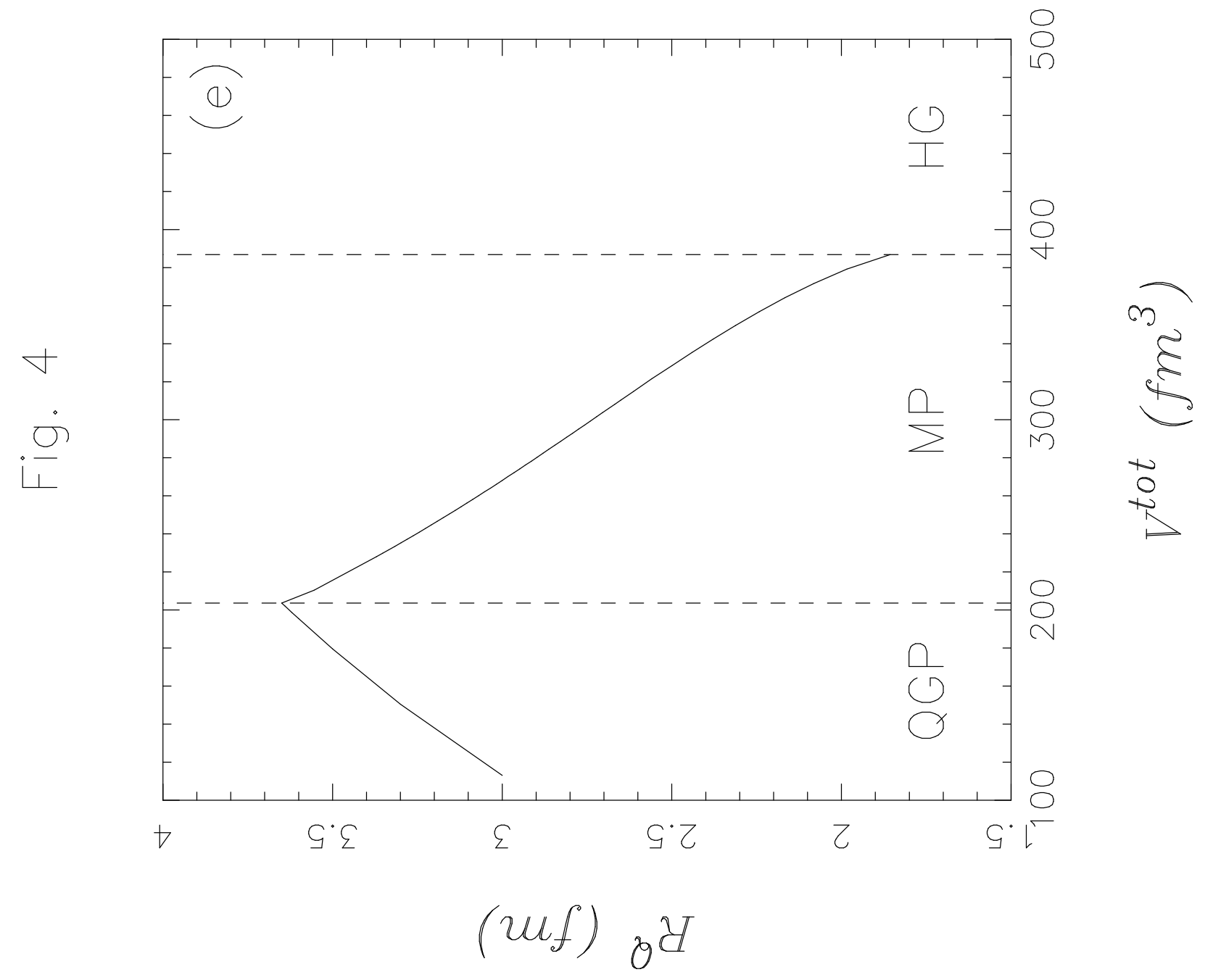

72 да ән แ!g-5นо人 


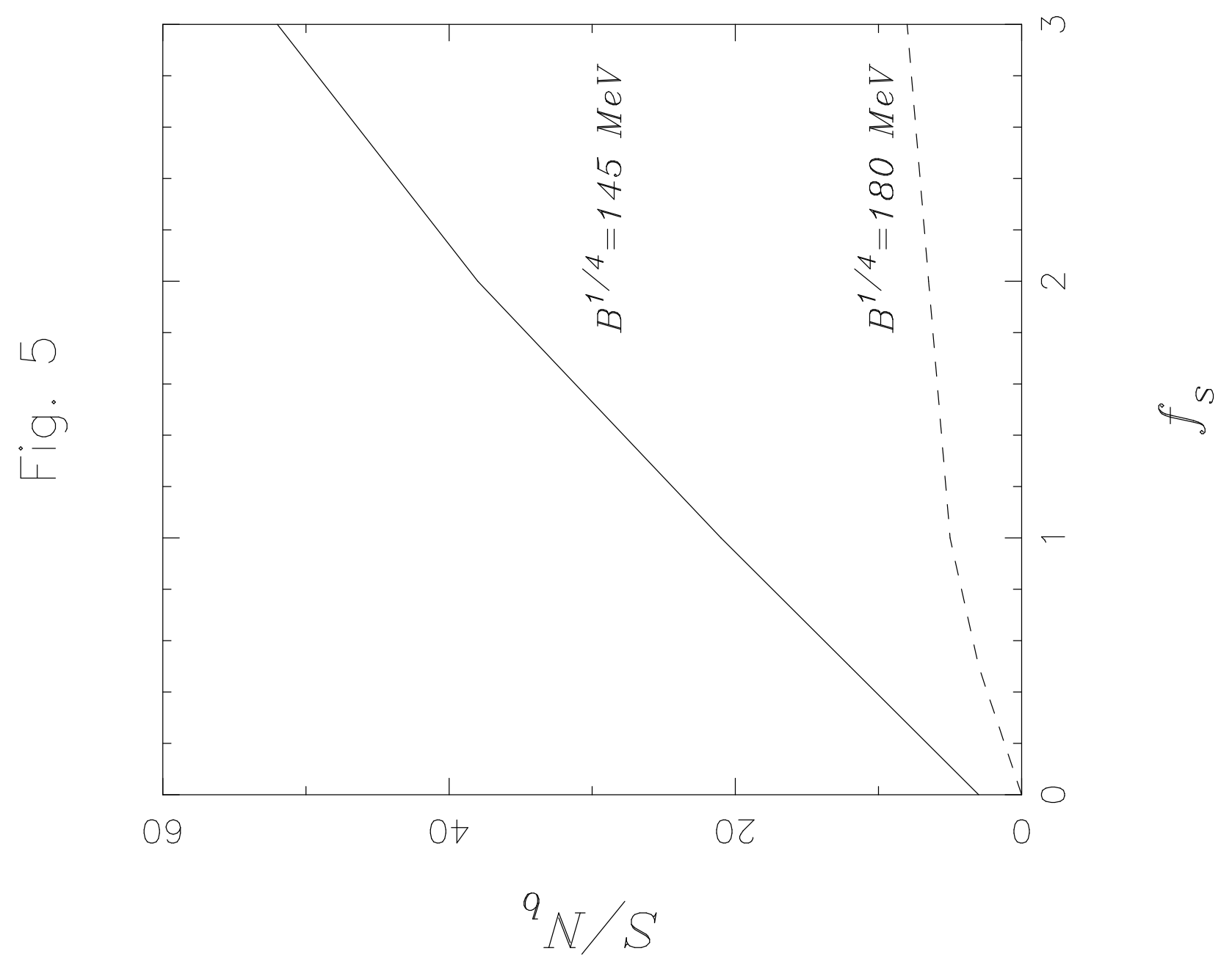

270 дә ән แ!g-6นо人 